\title{
Metabolomic profiling in evaluation of cultivated oat species with different ploidy level
}

\author{
Igor Loskutov, Tatiana Shelenga ${ }^{*}$, Elena Blinova, Alexandr Gnutikov, Alexey Konarev \\ Federal Research Center N.I. Vavilov All-Russian Institute of Plant Genetic Resources (VIR), \\ 42, 44 Bol'shaya Morskaya Street, St. Petersburg 190000, Russia
}

\begin{abstract}
The article presents biochemical characteristics identified during the analysis of metabolomic profiles. The object of this study was the landraces of cultivated oat species Avena strigosa Schreb., A. abyssinica Hochst. and A. sativa $\mathrm{L}$. with different ploidy levels from the global collection of the Department of Genetic Resources of Oats, Rye, and Barley of VIR. Metabolic profiling was performed using an Agilent 6850 gas chromatograph (Agilent Technologies, USA). The main task of this evaluation was determination of differences between cultivated oat species of diploid, tetraploid and hexaploid ploidy levels according to their metabolomic spectra. These spectra reflect the metabolic state of genotypes of various ecological and geographical origin. The investigation touched on the most important groups of metabolites important for the resistance formation nutritional, medicinal and dietary benefits. Particular, attention was paid to biologically active compounds that determine the functional value of daily human foods - phenolic compounds, free amino acids and sugar acids. The most informative indicators, which separate the oat with different ploidy levels were: isofucosterol, xylitol, MAG-1 18: 0, linolenic, undecylic, threonic, glutamic and methylmalonic acids.
\end{abstract}

\section{Introduction}

Recently, in addition to grain productivity, quality indicators of grain have become increasingly important. The traditional directions of breeding grain crops are the increasing content of protein, lysine, starch. Nowadays the dietary properties of grain are also in demand. In addition to protein, the grain crops are rich in other compounds, especially, essential fatty acids and antioxidants. Oats (Avena sativa L.) is a cereal crop grown mainly for feed and food purposes. Oats contain valuable nutrients such as minerals, lipids, proteins, vitamins, fiber, unsaturated fatty acids, as well as various biochemicals that play role in the prevention of such widespread diseases as cardiovascular disorders, colon cancer, type II diabetes and many others [1-3].

Currently, oat supplements can be found in a variety of daily foods such as breakfast cereals, dairy and meat products, breads, and baby food; however, oat-based drinks (often named as oat milk) have recently been introduced and are becoming the optimal functional

\footnotetext{
Corresponding author: t.shelenga@vir.nw.ru
} 
food for their nutritional and prebiotic potential. The fiber components ( $\beta$-glucans) found in oats are known for their beneficial effects on human health. As a result of the analysis of metabolomic profiles in this product, important classes of compounds were identified: flavonoids, phenolic alkaloids (avenanthramides), amino acids and steroids, which allows us to talk about the future development of cereal-based drinks production [3]. The above mentioned biologically active compounds of oat grain meet the requirements for the functional components of the human diet $[4,5]$.

The Russian Federation is the leader in the world in acreage area under oats and in grain production. For various reasons, the acreage area under oats is decreasing, and the demand for grain is growing. In this regard, the role of innovative breeding becomes more important. When creating new varieties, first of all, attention is paid to increasing productivity and resistance to unfavorable environmental factors in order to obtain stable yields and meet the needs for grain of feed and food quality. Well-studied accessions that are sources or donors of economically valuable traits are most often used as a initial material for breeding. Landraces and developed varieties can be also used as initial breeding material. In the doctrine of the source breeding material, N.I. Vavilov assigned an important role to local varieties, emphasizing the need to use the most productive and most valuable for adaptability forms [6]. In addition, the breeding value of the initial material is determined by the characteristics that provide resistance to biotic and abiotic environmental factors [7].

At the same time, the metabolomic profiles of caryopses of various oat species can represent the most complete biochemical characteristic, presence or absence of certain metabolites, which makes it possible to identify various species, subspecies, and individual oat accessions, representing a kind of biochemical "finger print". The features of the metabolomic profile characterize the biochemical phenotype formed under the influence of the genotype and environmental conditions [8]. Metabolic profiling at different stages of plant development makes it possible to study the biochemical composition in ontogenesis, on different agrophones and growing conditions, as well as in connection with the variety (specificity) of seeds [9-12].

As a result of metabolic profiling investigation in different oat species, the content of metabolites which decreased in the process of domestication or by which wild species differ from the varieties were identified. It is assumed that this may be associated with the formation of adaptability traits, in particular, with resistance to diseases and pests, abiotic environmental factors [13]. A number of properties specific for adaptation can be lost in the process of breeding highly specialized narrow-linear modern varieties of an intensive type, since this process is accompanied by a decrease in the genetic polymorphism of breeding varieties in comparison with the characteristics of population composition of wild species and local varieties created as a result of folk breeding [14-16]. During studying the metabolomic spectra of caryopses in plants susceptible to Fusarium infection, correlations between infection's indicators and biochemical parameters were revealed. It was found that high-protein forms of oats are less affected by Fusarium and accumulate less toxins: they are more adaptive to biotic stress [17].

The study of metabolite's profiles of living objects makes it possible to assess the effect of genetic modifications, biotic and abiotic stressors on them, and also facilitates the assessment of components with low concentrations in combination with high biological activity, such as phenolic compounds, polyols, free amino acids, phytosterols and a number of organic acids [13, 18-20]. Thus, metabolomic profiling is a promising approach for identifying the links between biochemical parameters and genetic characteristics of cereals and opening up new opportunities for targeted breeding for quality [21].

Analysis of the metabolic spectra of plant objects is a fairly new line of research in the field of omix studies. Using this method, we tried to determine how informative it is and how it allows to reliably identify the genetic diversity of any genus, including Avena L. In our 
previous studies, we obtained data that allowed us to statistically separate individual wild and cultivated oat species [22]. In addition, we succeeded in identifying peculiarities of metabolomic spectra in Avena sativa L. subspecies (covered and naked) [23]. We have identified statistical differences in the intraspecific diversity of varieties with different breeding levels and different geographic origins, where the history of oat breeding dates back to the end of the 19th century and achieved significant success in the 1920-1930s and at the beginning of the 20th century [24].

N.I. Vavilov All-Russian Institute of Plant Genetic Resources (VIR) has an extensive collection of the genus Avena L. (14 thousand accessions), including wild species and the world diversity of cultivated species, which are represented by landraces-populations, primitive (obsolete) breeding, modern breeding varieties and breeding lines. All this material is represented by complete geographic diversity from all continents of the world. A comprehensive study of this diversity makes it possible to select the source material for breeding and send it to the leading breeding centers of the Russian Federation for breeding work $[7,25]$.

The purpose of this research was to identify differences between accessions of cultivated oat species of diploid, tetraploid and hexaploid ploidy levels for metabolomic spectra.

\section{Materials and Methods}

The evaluation took a unique intraspecific diversity of 30 landraces of cultivated oat species: diploid A. strigosa Schreb., tetraploid A. abyssinica Hochst. and hexaploid A. sativa L., collected by N.I. Vavilov and his associates in 1920-1930s (Tab. 1). This material is characterized by increased adaptability to changing cultivation conditions. The conducted field phenotyping of all studied species using the methodological guidelines of the VIR [26] demonstrated a significant diversity in the main economically valuable traits, as a result, accessions with increased characteristics of the studied traits were identified. The grain from the propagated accessions was used to carry out metabolic profiling and to isolate accessions with high biochemical characteristics. In addition, to compare metabolomic spectra in the dynamics of plant development, metabolomic profiles were obtained in 10-day-old seedlings of local varieties of all studied species.

Table 1. Studied landraces of cultivated oat species.

\begin{tabular}{|c|c|c|}
\hline Species & Origin & Number of accessions \\
\hline A. strigosa Schreb. & Portugal & 5 \\
\hline " & Spain & 5 \\
\hline A. abyssinica Hochst. & Ethiopia & 10 \\
\hline A. sativa L. & Italy & 5 \\
\hline " & Portugal & 3 \\
\hline " & Spain & 1 \\
\hline " & Greece & 1 \\
\hline & TOTAL & $\mathbf{3 0}$ \\
\hline
\end{tabular}

Sample preparation and metabolomic profile analysis. Metabolomic profile was analysed using gas chromatography with mass spectrometry according to a protocol [27]. $10 \mathrm{~g}$ from a mix of harvesting seeds of each accession were milled by the preparation mill Lab. mill-I (Labor Muszeriparimuv, Hungary), then 0,2 $\mathrm{g}$ of powder were homogenized with appropriate amounts of methanol at the ratio of $1: 10$. The resulting extract was centrifuged at 14,000 rpm for $10 \mathrm{~min}$ and $100 \mu \mathrm{L}$ was evaporated using CentriVap Concentrator (Labconco, USA). 20 $\mu \mathrm{L}$ of bis(trimethylsilyl)acetamide reagent was added to the solid residue and kept for $15 \mathrm{~min}$ under $100^{\circ} \mathrm{C}$ in a DigiBlock digester (Laboratory devices, inc., USA). Tricosane (normal hydrocarbon $\mathrm{C} 23 \mathrm{H} 48$ ) was added as an internal standard in the following concentrations: 
$1 \mu \mathrm{g} / \mu \mathrm{L}$. The analysis was performed on an HP-5MS capillary column (5\% phenyl, $95 \%$ methylpolysiloxane, $30.0 \mathrm{~m}, 250.00 \mu \mathrm{m}, 0.25 \mu \mathrm{m}$ ) using an Agilent 6850 gas chromatograph with a quadrupole mass selective detector Agilent 5975B VL MSD (Agilent Technologies, USA). Conditions of the analysis: helium flow $-1.5 \mathrm{~mL} / \mathrm{min}$; heating program - from $+70^{\circ} \mathrm{C}$ up to $+320^{\circ} \mathrm{C}$; heating rate $-4^{\circ} \mathrm{C} / \mathrm{min}$; detector temperature $-+250^{\circ} \mathrm{C}$; injector temperature $-+300^{\circ} \mathrm{C}$; injection volume $-1.2 \mu \mathrm{L}$, with the splitless flow. The peaks were registered on an Agilent 5975B mass selective detector in the total ion recording mode with a frequency of 1.8 scans per second within the range of $50-850 \mathrm{~m} / \mathrm{z}$. The recording of a chromatogram started after $4 \mathrm{~min}$ required for solvent removal and went on for $62 \mathrm{~min}$. There were three analytical replications of the analysis, average data was obtained and used for the statistical processing.

Deconvolution and metabolite identification were processed with AMDIS (Automated Mass spectral Deconvolution and Identification System). Chemical compounds were identified by their mass spectra and retention indices using several databases. In addition to NIST2010 (National Institute of Standards and Technology, USA), the libraries of the Research Park of St. Petersburg University and the Komarov Botanical Institute of the Russian Academy of Sciences were also applied [28]. These last two databases were developed as the result of previous standard-based chemical determination performed at St. Petersburg University and the Botanical Institute of the Russian Academy of Sciences. The retention indices (RI) were estimated by the calibration of saturated hydrocarbons with the number of carbon atoms ranging from 10 to 40. A semi-quantitative assay of the metabolite profiles was performed by calculation of the total ion peak areas with the internal standard method using UniChrom software (New Analytical Systems, Belarus, www.unichrom.com). The relative content of biochemical components is expressed in ppm DW ( $\mu \mathrm{g} / \mathrm{g} / \mathrm{dry}$ matter').

\section{Results and Discussion}

As a result of the evaluation, it was found that the metabolome profile of oat kernels, on average, consisted of more than 350 components, of which over 100 were identified. The identified components represented the following groups of compounds: organic acids, free amino acids, nucleosides, free fatty acids, acylglycerols (MAG-1 C16: 0, MAG-1 C18: 0, MAG-2 C18: 3, MAG-2 C18: 2, DAG), polyols, phytosterols, phenol-containing compounds (PCC), mono and oligosaccharides. A comparative detailed analysis of the main groups of metabolites of caryopses accessions of cultivated oat species with different levels of ploidy - A. strigosa (diploid), A. abyssinica (tetraploid), A. sativa (hexaploid) showed that diploid accessions have the highest content of free amino acids, polyols, monosaccharides, glycosides, acylglycerols. In tetraploid - organic acids, PCC phenolic compound and oligosaccharides. Hexaploid - free fatty acids, phytosterols.

Metabolic profiles (MP) of accessions of different ploidy differed in qualitative and quantitative composition. In diploid and hexaploid accessions, malic and gluconic acids prevailed in the group of organic acids, while galacturonic and malic acids - in tetraploid accessions. Azelaic and ribonic acids were absent in the MP of diploid oats, and azelaic acid in tetraploid oats (Fig. 1). 


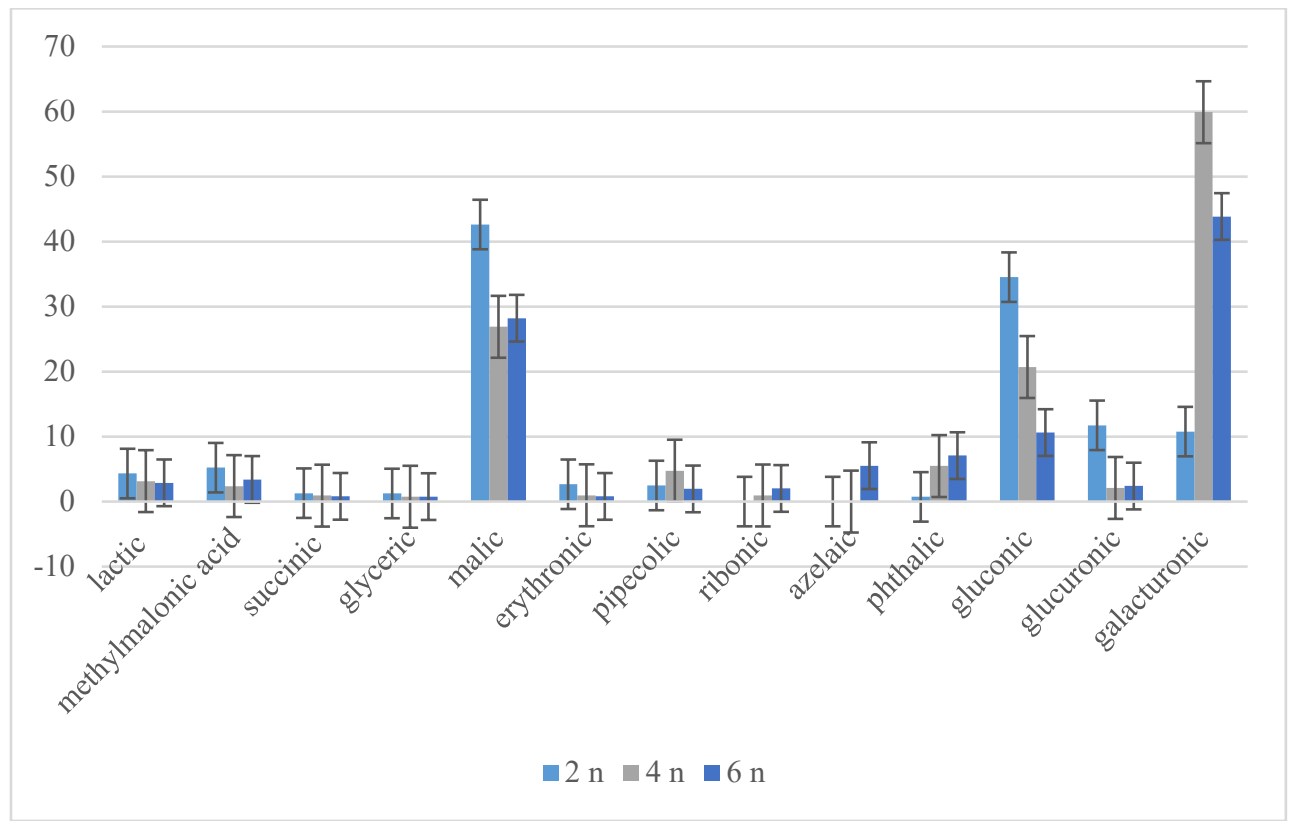

Fig. 1. Representation of organic acids in the MP in oat kernels of different ploidy.

In the group of free amino acids oat's MP, glycine and asparagine predominated; the highest values of these acids were observed in diploid accessions. In tetraploids, in addition to the above mentioned, high values of the non-protein pipecolic amino acid were noted (Fig. 2).

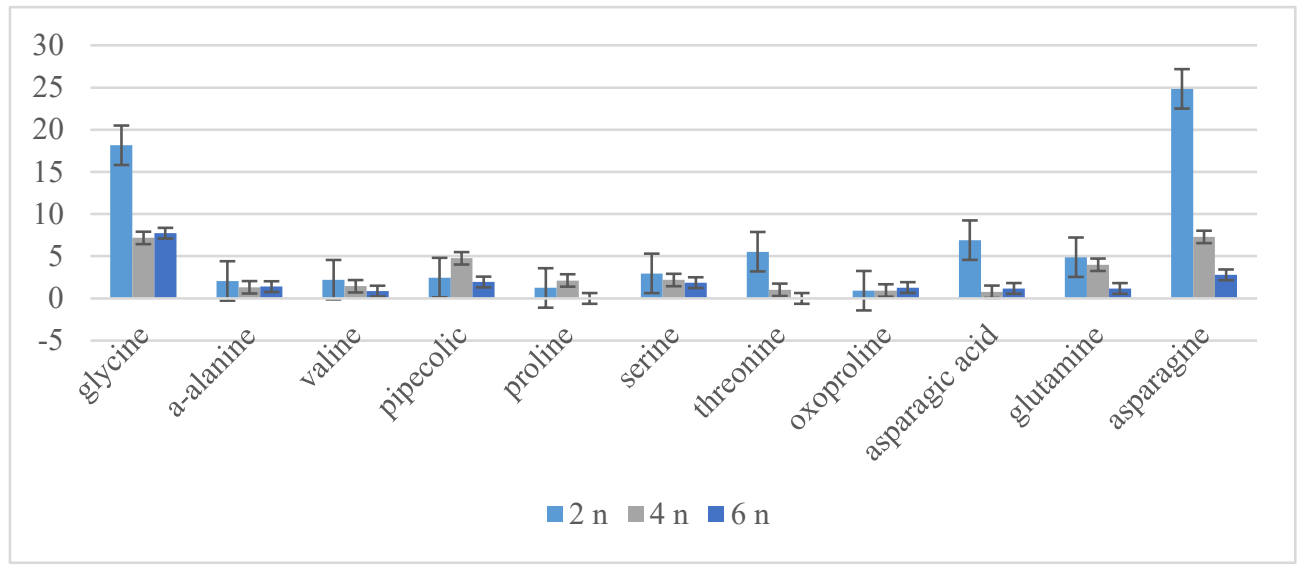

Fig. 2. Free amino acids MP in oat caryopses of different ploidy.

Polyols in diploid and tetraploid accessions were represented by glycerol and myoinositol. In caryopses of hexaploid oats, the content of glycerol, dulcitol, myo-inositol, and sorbitol was found to be equally high (Fig. 3). 


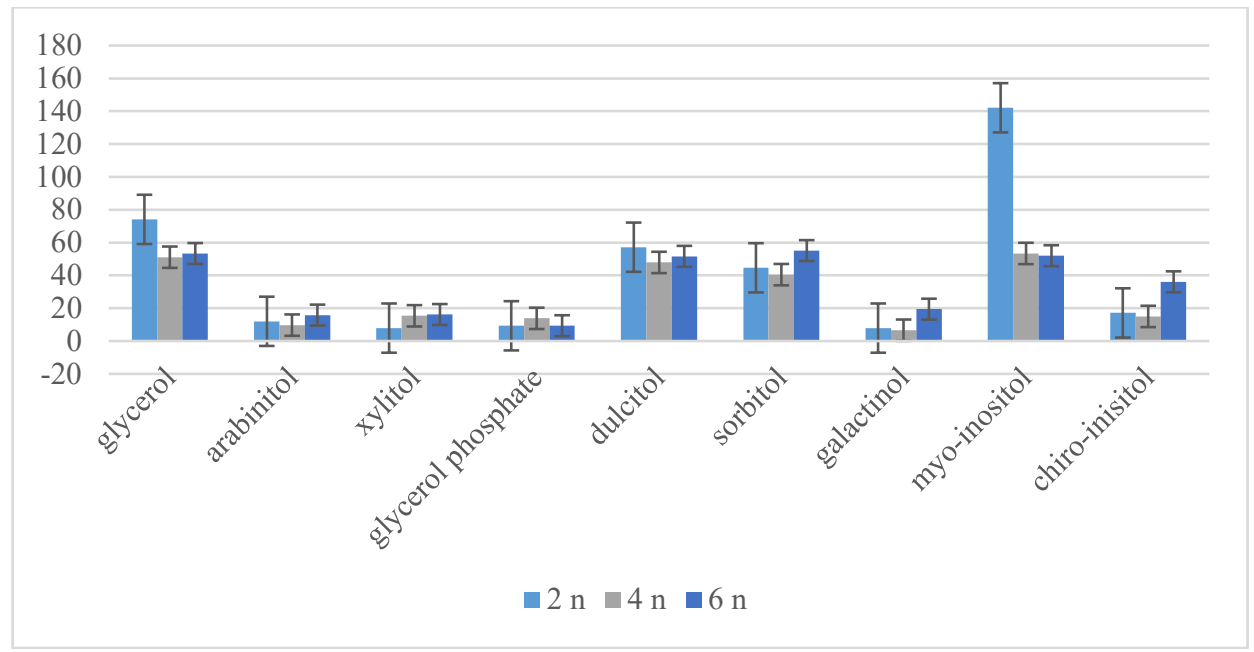

Fig. 3. Polyols MP in oat caryopses of different ploidy.

Phytosterols in MP are represented by isofucosterol and sitosterol. Of these, sitosterol was found to be the most typical for all studied accessions; its highest content was determined for hexaploid oats (Fig. 4).

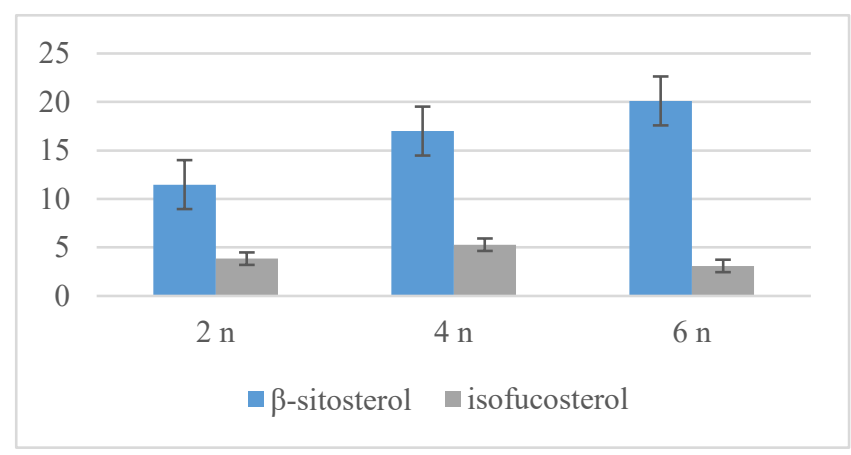

Fig. 4. Phytosterols MP in oat caryopses of different ploidy.

In the group of monosaccharides in the MP of oats, in addition to glucose and fructose, high levels of ribose were noted. Arabinose was identified in hexaploid oats, and gulose in tetra- and hexaploid accessions (Fig. 5). 


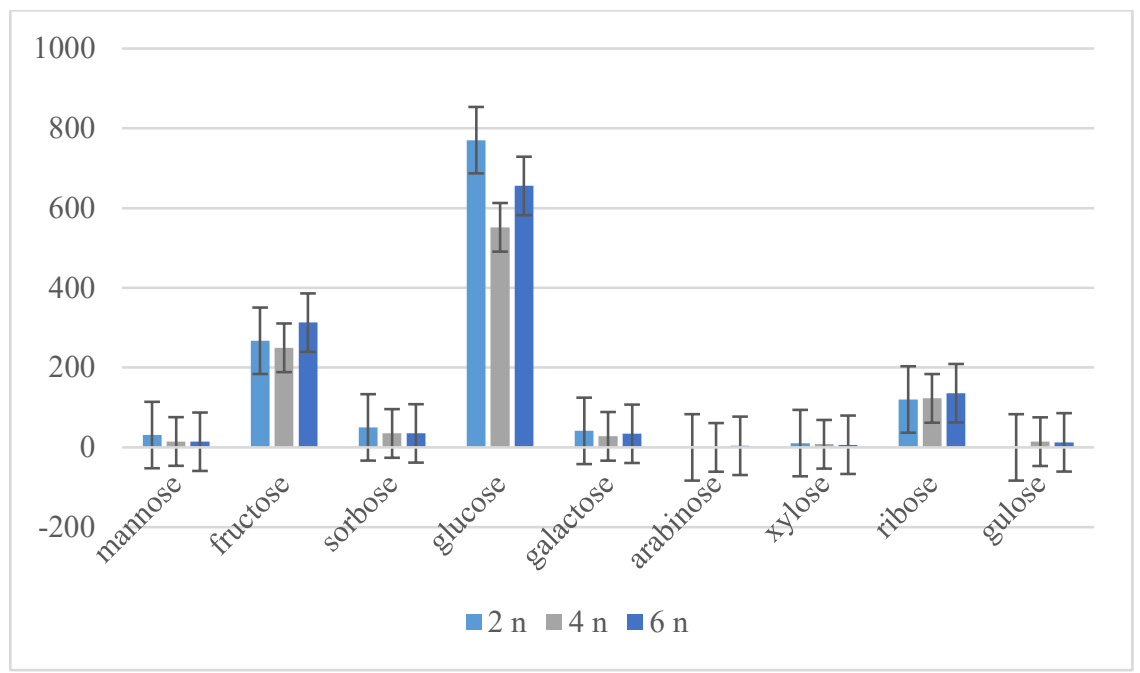

Fig. 5. Monosugar MP in oat caryopses of different ploidy.

In all the oat's accessions, oligosaccharides such as sucrose and turanose prevailed in the MP (Fig. 6).

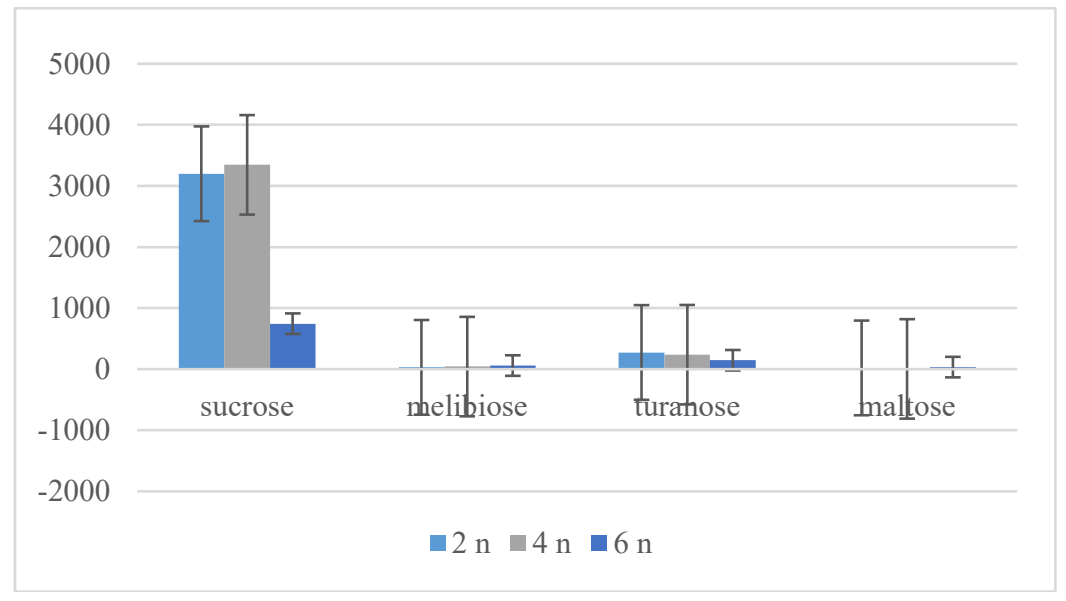

Fig. 6. Oligosugars MP of in oat caryopses of different ploidy.

Three fatty acids were predominated in the MP: palmitic, vaccenic, and linoleic. Pelargonic acid was not detected in the MP of di- and tetraploids (Fig. 7). 


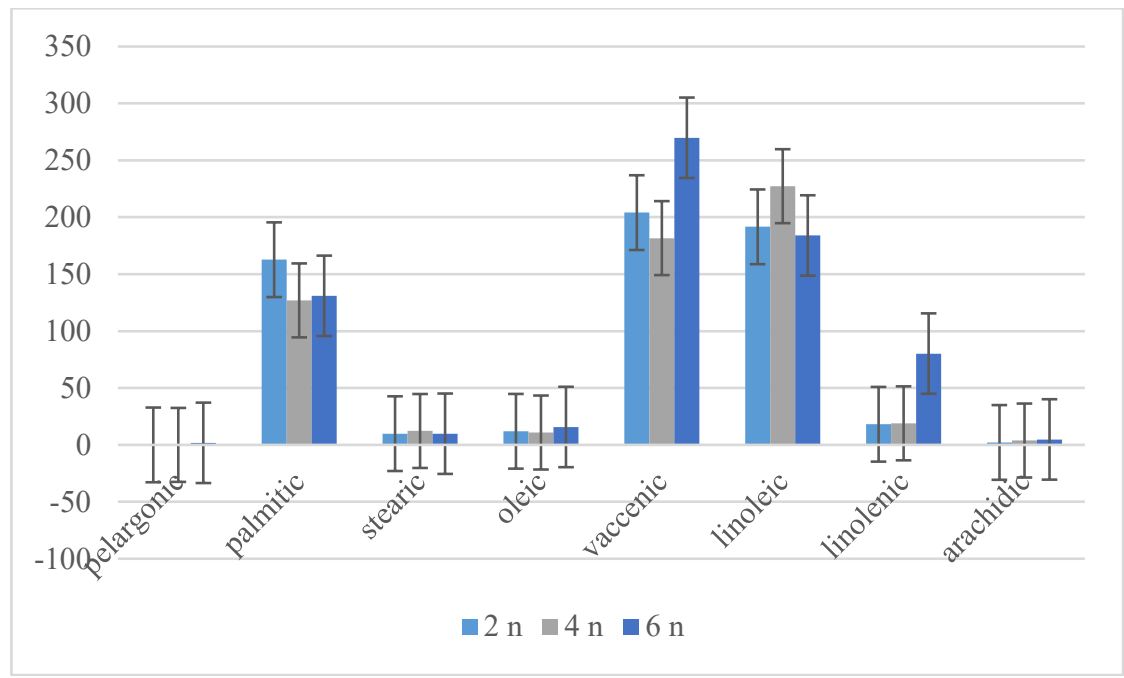

Fig. 7. Free fatty acids MP in oat kernels of different ploidy.

In the acylglycerol's group, MAG1 16: 0 and MAG-218: 2 were distinguished by the highest content (Fig. 8).

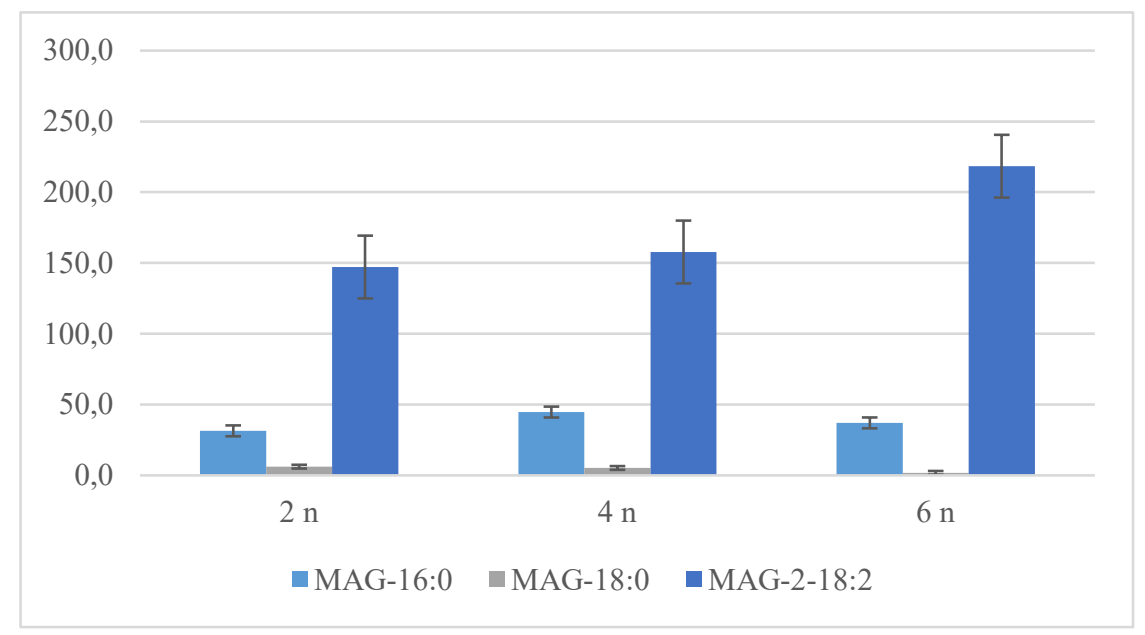

Fig. 8. Acylglycerols MP in oat caryopses of different ploidy.

The group of phenol-containing compounds (PCC) is represented by kaempferol, benzoic and nicotinic acids with a predominance of kaempferol (Fig. 9). 


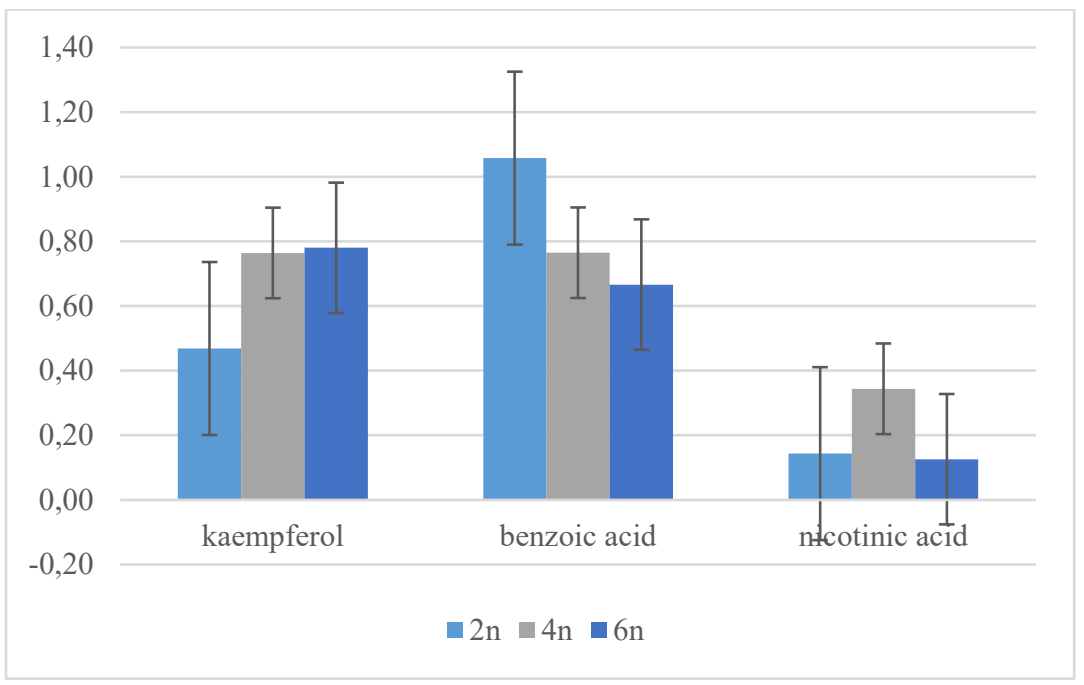

Fig. 9. Phenol-containing compounds MP in oat caryopses of different ploidy.

Thus, the diploid species A. strigosa was distinguished by an increased content of glucuronic, malic, gluconic, aspartic palmitic, vaccenic, benzoic acids, glycine, asparagine, dulcitol, glucose, and turanose. The tetraploid species A. abyssinica - galacturonic, pipekolinic, linoleic, nicotonic acids, glycerol, gulose, sucrose, MAG1 16: 0. The hexaploid species A. sativa is methylmalonic, azelaic, phthalic, vaccenic, oleic, linoleic acids, glycerol, dulcitol, myo-inositol, sorbitol, sitosterol, arabinose, maltose, melibiose, MAG-218: 2, kaempferol.

At the next stage of the research work, a comparative analysis of the metabolomic profiles of oat caryopses of different ploidy degrees $(2,4,6 \mathrm{n})$ and their weekly seedlings was carried out. The purpose of the experiment was to study changes in the metabolic network at different stages of plant development using the example of wild forms of oats. As a result of the experiment, the forms with the maximum indicators of the compounds responsible for the plant's resistance to stress and nutritional value were identified.

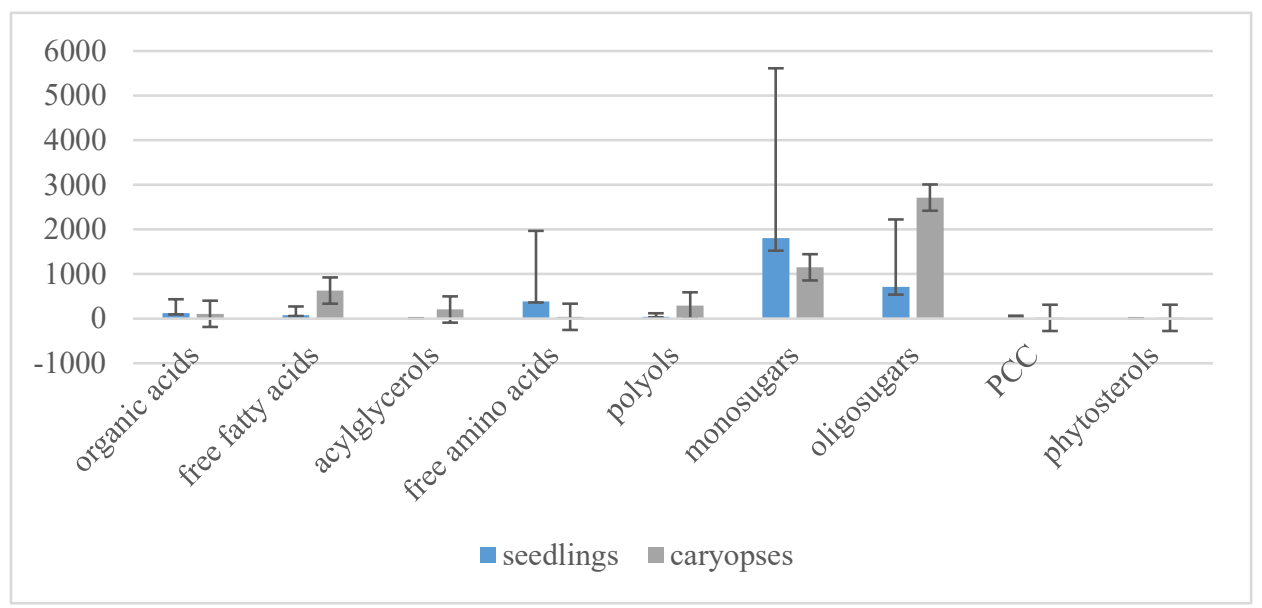

Fig. 10. Different groups of metabolites in oat's seedlings and caryopses.

It was found that the total content of organic acids, free amino acids in seedlings was higher, and the qualitative composition was more varied in comparison with oat caryopses. 
In the first case, acids of the Krebs cycle (AKC) and shikimate pathway (malic, aconitic, quinic acids) dominated, in the second - malic and uronic acids - oxidation products of the corresponding monosaccharides and components of plant biopolymers, which are valuable indicators when choosing raw materials for products. functional human nutrition. Free amino acids of seedlings and caryopses were mainly represented by neutral, non-polar and polar forms. In seedlings, asparagine prevailed among free amino acids; its content was much (about 10 times) higher than that of other free amino acids. Asparagine is closely related to TCA, its precursor is oxaloacetate. The caryopses contain glycine, one of the most common amino acids, which is part of proteins and biologically active compounds. Pipecolic acid, a factor of plant resistance to stress, has been identified only in caryopses (Fig. 10).

The total content of monosaccharides was higher in seedlings than in caryopses. The quality composition was almost the same. The dominant compound is glucose, one of the most widespread monosaccharides not only in plants, but also in any living organism.

Quantitative indicators and qualitative composition of free fatty acids, polyols, oligosaccharides were higher and more diverse in oat caryopses. The group of free fatty acids was dominated by vaccenic, linoleic and palmitic acids, polyols - isomers of inositol, oligosaccharides - sucrose. In oat kernels, the amount of acylglycerols was higher and the composition was more varied (monoacylglycerol of linoleic acid MAG-2 18: 2 prevailed). In the tissues of seedlings, only one monoacillicerol of palmitic acid (MAG-1 16: 0) was identified.

The indices of PCC were practically the same for caryopses and oat seedlings, but they were represented by different compounds. In seedlings - shikimic and quinic acids, in caryopses - methylated phenol, which is part of vitamin E, $\alpha$-tocopherol. In addition to the aforementioned groups of compounds, phytosterols with a predominance of $\beta$-sitosterol and adenosine nucleoside were identified in caryopses.

Seedlings of diploid accessions were distinguished by a higher content of free amino acids, essential amino acids (valine, lysine, leucine, threonine), tetraploid ones - asparagine, free fatty acids, sugar acids, myo-inositol, mono and oligosaccharides, hexaploid - organic acids, malic and galacturonic acids, galactinol, phenolic compounds, diploid and tetraploid monoacylglycerol of palmitic acid, tetraploid and hexaploid - polyols (Fig. 11).

The total content of organic acids, phytosterols, adenosine, in oat grains of different ploidy was almost the same, but they differed in the number of individual compounds. In the diploid accessions of caryopses, there was an increased content of free amino acids, polyols, monosaccharides, glyceric, gluconic acids, glycine, glutamine, asparagine, inositol isomers, tetraploid - uronic, linoleic pipicolic acids, a-tocopherol, hexaploid - fatty acids: vaccenic, linolenic acids, monoacylglycerol of palmitic acid, di- and tetraploid - oligosaccharides, tetra- and hexaploid - phytosterols (Fig. 12, 13). 


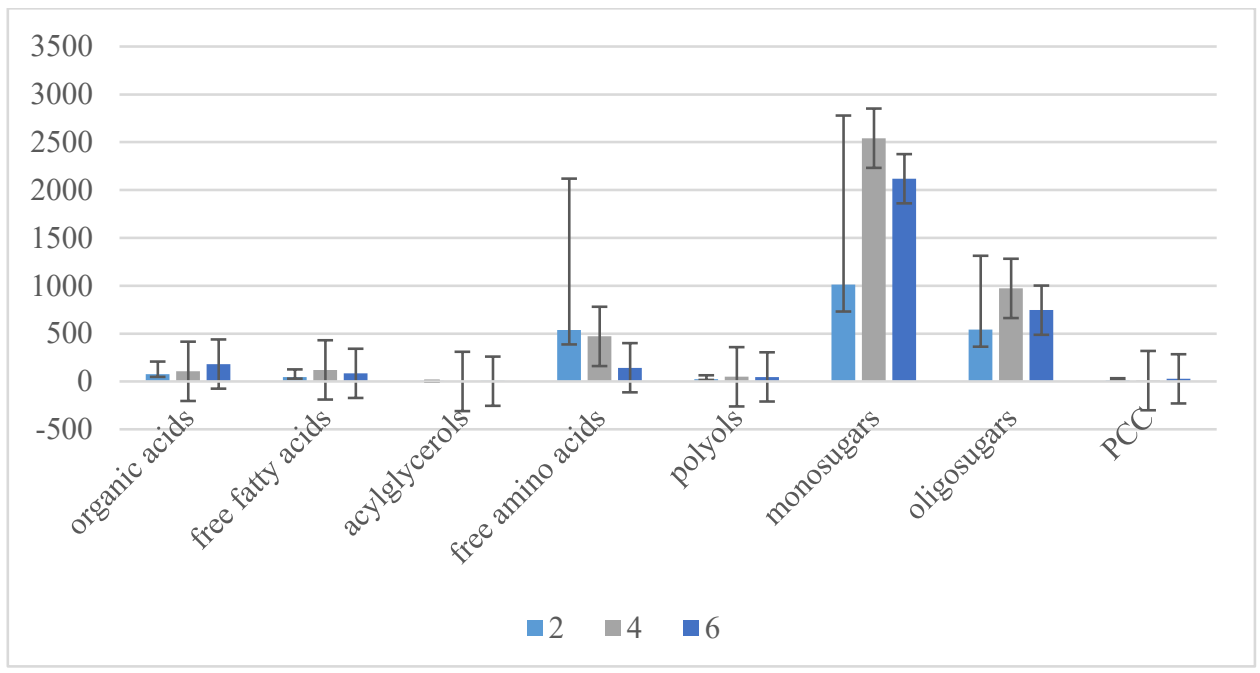

Fig. 11. Different groups of metabolites in oat's seedlings and caryopses with different levels of ploidy.

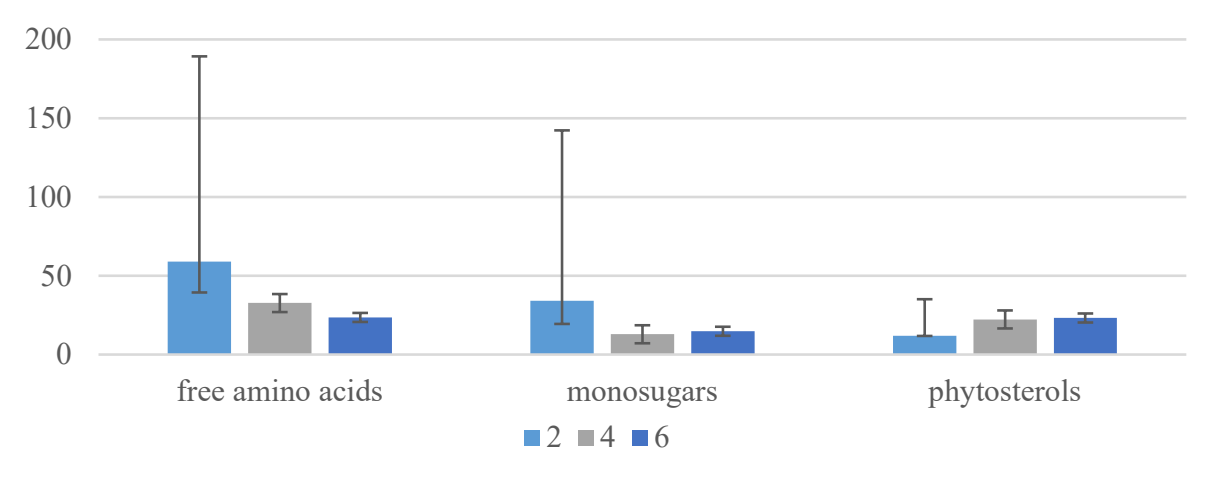

Fig. 12. Metabolic profile characters in oat's caryopses with different levels of ploidy.

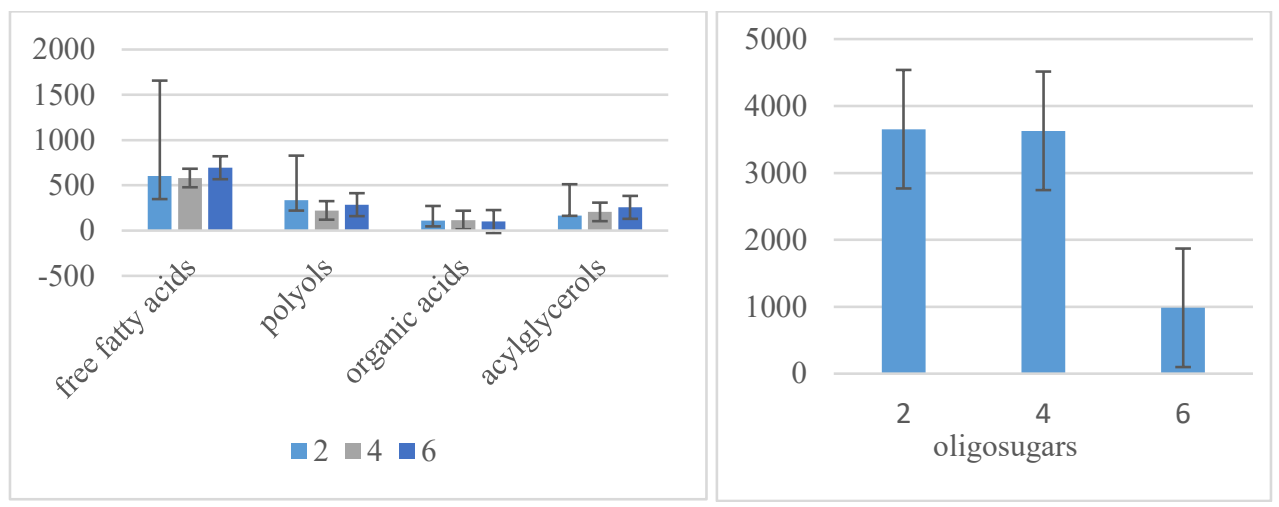

Fig. 13. Metabolic profile characters in oat's caryopses with different levels of ploidy.

Thus, seedlings and caryopses of oats at the stage of full ripeness differed both in the qualitative composition of metabolomic profiles and in the content of individual compounds 
and groups of metabolites, which is associated with different intensities of primary and secondary metabolites at different stages of oat development.

Oat seedlings were distinguished by higher values of quinic and shikimic acids, substances by the presence of which it is possible to assess the activity of the synthesis of biologically active compounds, aspararin, an amino acid important for the normal functioning of the human nervous system, organic acids necessary for the functional stability of the gastrointestinal tract, monosaccharides, the main sources of energy and components of most metabolic processes in the human body. The grain was found to be more significant indicators of pipecolic acid, acylglycerols, oligosaccharides, polyols, including galactinol, involved in the system of adaptation to unfavorable environmental factors; adenosine, which has an antiinflammatory effect and has a positive effect on patients suffering from chronic diseases of the cardiovascular system; glycine and inositol isomers necessary to maintain the stable functioning of the central nervous system; free fatty acids, $\alpha$-tocopherol, phytosterols, uronic acids, components that reduce the concentration of cholesterol in the blood and normalize metabolism in the human body as a whole.

\section{Conclusions}

Compounds responsible for nutritional value were better represented in diploid accessions of caryopses. For example, glyceric acid is an intermediate product of glycolysis and biosynthesis of a number of amino acids, glucuronic acid, the building blocks of human connective tissue, in addition, glycine has a calming, antidepressant effect, reduces the severity of side effects of antipsychotics, gluconic acid, which has a positive effect on metabolism in the body as a whole, aspartic acid is necessary for the normal functional state of the brain, benzoic acid, natural antiseptic, etc. Uronic acids, important for the normal functioning of the digestive system, were found in large quantities in tetra- and hexaploid forms; are part of biopolymers involved in the detoxification of harmful substances in the human body, phytosterols, which help to reduce blood cholesterol and have an anti-cancer effect, essential fatty acids, azelaic acid, which has anti-inflammatory properties, as well as substances associated with plant resistance to environmental stress (pipecolic acids and acylglycerols).

Seedlings of various degrees of ploidy were characterized not only by different indicators of nutritional value (essential amino acids, essential fatty acids, inositols, uronic acids, biologically active substances of a phenolic nature, etc.), but also by resistance to adverse conditions (tetra- and hexaploid accessions).

Thus, for almost all accessions, valuable biochemical indicators have been established that can be used in various breeding programs when creating varieties with a certain set of target compounds (food components) to create new products for therapeutic, prophylactic and functional nutrition of the population of the Russian Federation.

\section{Acknowledgements}

This work was supported by the Russian Foundation for Basic Research, project no. 17-0000340 (17-00-00336, 17-00-00337, 17-00-00338) and project no. 20-516-10002.

\section{Conflicts of Interest}

Declare conflicts of interest or state "The authors declare no conflict of interest." 


\section{References}

1. I.G. Loskutov, V.I. Polonskiy, Agric. Biol. 52, 646-657 (2017) doi: 10.15389/agrobiology.2017.4.646eng

2. V. Polonskiy, I. Loskutov, A. Sumina, Biol. Commun 65, 53-67 (2020) DOI: https://doi.org/10.21638/spbu03.2020.105

3. S. Bocchi, G. Rocchetti, M. Elli, L. Lucini, Ch-Y. Lim, L. Morelli, Food Res. Inter. 142, 110216 (2021) https://doi.org/10.1016/j.foodres.2021.110216

4. S. Leonova, A. Gnutikov, I. Loskutov, E. Blinova, K-E. Gustafsson, O. Olsson Proceedings on Applied Botany, Genetics and Breeding 181 (1), 30-47 (2020) DOI: 10.30901/2227-8834-2020-1-25-42

5. N.A. Shvachko, I.G. Loskutov, T.V. Semilet, V.S. Popov, O.N. Kovaleva, A.V. Konarev, Molecules 26, 2260 (2021) https:// doi.org/10.3390/molecules26082260

6. N.I. Vavilov, General plant breeding 1, 1042 (1935)

7. I.G. Loskutov, H.W. Rines L.In Avena, C. Kole (ed.), Cereals. Springer, Heidelberg, Berlin, New York, 1, 109-184 (2011) doi: 10.1007/978-3-642-14228-4

8. I.G. Loskutov, T.V. Shelenga, A.V. Rodionov, V.I. Khoreva, E.V. Blinova, A.V. Konarev, A.A. Gnutikov, A.V. Konarev, Proceedings of the Latvian Academy of Sciences. Section B. Natural, Exact, and Applied Sciences 73 (6), 494-501 (2019) doi: 10.2478/prolas-2019-0076

9. G.N. Smolikova, A.L. Shavarda, I.V. Alekseychuk, V.V. Chantseva, S.S. Medvedev, Vavilov Journal of Genetics and Breeding 19 (1), 121-127 (2015)

10. N. Schauer, A.R. Fernie, Trends Plant Sci. 11 (10), 508-516 (2006) https://doi.org/10.1016/j.tplants.2006.08.007.

11. P. Langridge, D. Fleury, Trends Biotechnol., 29 (1), 33-40 (2011) https://doi.org/10.1016/j.tibtech.2010.09.006

12. D. Balmer, V. Flors, G. Glauser, B. Mauch-Mani, Front Plant Sci. 4, 82 (2013) https://doi.org/10.3389/fpls.2013.00082.

13. S. Žilić, V.H. Šukalović, D. Dodig, et al., J Cereal Sci. 54(3), 417-424 (2011) https://doi.org/10.1016/j.jcs.2011.08.006.

14. V. Shulaev, D. Cortes, G. Miller, R. Mittler, Physiologia Plantarum 132, 199-208 (2008)

15. J. Perkowski, K. Stuper, M. Buúko, T. Góral, A. Kaczmarek, H. Jeleñ, J. Cereal Sci. 56, 544-551 (2012)

16. J. Gu, L. Jing, X. Ma, Z. Zhang, Q. Guo, Y. Li, J. Nutritional Biochemistry 26, 15091519 (2015)

17. I.G. Loskutov, T.V. Shelenga, A.V. Konarev, V.I. Khoreva, F.L. Shavarda, E.V. Blinova, A.A. Gnutikov, Agric. Biol. 54 (3), 575-588 (2019) doi: 10.15389/agrobiology.2019.3.575eng

18. I. Björck, E. Östman, M. Kristensen, et al., Trends Food Sci Technol. 25(2), 87-100 (2012) https://doi.org/10.1016/j.tifs.2011.11.005.

19. B. Khakimov, S. Bak, S.B. Engelsen, J Cereal Sci. 59 (3), 393-418 (2014) https://doi.org/10.1016/j.jcs.2013.10.002.

20. Y. Kokubo, M. Nishizaka, N. Ube, et al., Biosci Biotechnol Biochem 81(3), 431-440 (2017) https://doi.org/10.1080/09168451.2016.1256758.

21. A.R. Fernie, N. Schauer, Trends Genet, 25 (1), 39-48 (2009) https://doi.org/10.1016/j.tig.2008.10.010. 
22. I.G. Loskutov, T.V. Shelenga, A.V. Konarev, A.L. Shavarda, E.V. Blinova, N.I. Dzubenko, Rus J Genet Appl Res. 7(5), 501-508 (2017) https://doi.org/10.1134/s2079059717050136

23. I.G. Loskutov, T.V. Shelenga, A.V. Konarev, Yu.I. Vargach, E.A. Porokhovinova, E.V. Blinova, A.A. Gnutikov, A.V. Rodionov, Ecological genetics 18 (1), $27-41$ (2020) https://doi.org/10.17816/ecogen 12977

24. I.G. Loskutov, T.V. Shelenga, A.V. Konarev, V.I. Khoreva, Yu.A. Kerv, E.V. Blinova, A.A. Gnutikov, A.V. Rodionov, L.L. Malyshev, Proceedings on Applied Botany, Genetics and Breeding, 182 (2021) (in press)

25. I.G. Loskutov, Oats (Avena L.). Distribution, taxonomy, evolution and breeding value, 336 (VIR, St. Petersburg, 2007. p.

26. I.G. Loskutov, O.N. Kovaleva, E.V. Blinova Methodical guidelines for the study and preservation of the world collection of barley and oats, 63 (VIR, 2012)

27. I.N. Perchuk, T.V. Shelenga, M.V. Gurkina, E.V. Miroshnichenko, M.O. Burlyaeva, Molecules, 25(17), 3778 (2020) DOI:10.3390/molecules25173778

28. O. Shtark, D. Romanyuk, M. Kliukova, I. Tikhonovich, V. Zhukov, R. Puzanskiy, A. Shavarda, G. Avdeeva, V. Yemelyanov, A. Kirpichnikova, M. Shishova, Plants 10 (6), 1033 (2021) https://doi.org/10.3390/plants10061033. 